\title{
Utilising Semantic Technologies for Decision Support in Dementia Care
}

\author{
Taha Osman, Suganth Rmaswamy, Sawsan \\ Mahmoud \\ CIB, School of Science and Technology, Nottingham \\ NH11 8NS, UK \\ \{taha.osman, suganth.ramaswamy, \\ sawsan.mahmoud\}@ntu.ac.uk
}

\author{
Mahmoud Saeed \\ 24 Maple Leaf Drive, John Black Day Hospital, \\ Birmingham B37 7JB, UK \\ mahmoud.saeed@bsmhft.nhs.uk
}

\begin{abstract}
The main objective of this work is to discuss our experience in utilising semantic technologies for building decision support in Dementia care systems that are based on the non-intrusive on the non-intrusive monitoring of the patient's behaviour. Our approach adopts context-aware modelling of the patient's condition to facilitate the analysis of the patient's behaviour within the inhabited environment (movement and room occupancy patterns, use of equipment, etc.) with reference to the semantic knowledge about the patient's condition (history of present of illness, dependable behaviour patterns, etc.). The reported work especially focuses on the critical role of the semantic reasoning engine in inferring medical advice, and by means of practical experimentation and critical analysis suggests important findings related to the methodology of deploying the appropriate semantic rules systems, and the dynamics of the efficient utilisation of complex event processing technology in order to the meet the requirements of decision support for remote healthcare systems.
\end{abstract}

Keywords- Semantic Web; Dementia; Decision Support; Remote Healthcare; Knowledge Inference; Context Awareness

\section{INTRODUCTION}

The number of people over the age of 60 is expected to rise from $33 \%$ of those aged between 15 and 60 in 2001 to $55 \%$ in early $2030 \mathrm{~s}$, and for the people over the age of 75 the figure is expected to rise sharply from 4.4 million in 2000 to 8.3 million in 2040 [1]. This sharp increase in aging population is exerting enormous pressure on the resources of healthcare and social services systems. Cognitive decline and the related diseases such as Dementia and Alzheimer's disease (AD) are becoming increasingly common in the geriatric population. Worldwide, 34 million people suffer from Dementia and in UK alone this figure is nearly 700,000 [2]. The cost of Dementia to the UK economy is approximately 17 billion GBP each year [3].

Dementia sufferers can be broadly categorized into two groups - those in their early stages of the disease and those in their later stages [4]. For those in their later stages of the disease, personal care is inevitable. However, for those in the early stages of the disease, remote healthcare systems can complement and in some instances substitute personal care.

In the present scenario, care is given to dementia sufferers by NHS in the form of personal attendants such as nurses, social workers etc. This is one of the major factors responsible for the huge cost associated with dementia care. Reducing the amount of personal care devoted to early dementia sufferers by means of the remote monitoring of their condition not only reduces the pressure on NHS resources, but also promotes good quality independent living of the dementia sufferer.

Remote healthcare for dementia suffers has attracted a lot of investment. A large body of commercial and research efforts in the field concentrated mainly on memory loss, which is a main characteristic of dementia. Such systems offer guidance to the patients in their day-to-day activities such as reminding the patients to cook a meal, take medicine or even remind them to go to sleep [5][6]. This research subscribes to the more challenging efforts that deploy sensory devices to monitor the activities of daily living of early dementia suffers, and then analyse the sensory output to identify deviations from normal behavioural patterns that can indicate a deterioration in the some the conditions symptoms such as restlessness and wandering and subsequently advise intervention from the caregivers or the health clinicians.

This paper reports on our investigation into the utilisation of semantic technologies to build a context-aware Dementia Care Decision Support System (DCDSS), starting from knowledge elicitation from the domain experts and ontology engineering to knowledge inference. We devote special attention to the role of knowledge inference in the remote healthcare systems, and contribute to the understanding of the computational capacity, reasoning complexity, and interactivity dynamics with complex event streams that is necessary to fulfil the requirements for remote healthcare decision support. The rest of this paper is organised as follows: section 2 presented the motivation behind undertaking this research. Section 3 gives high-level view of our modelling approach and details the general system requirements. Building the decision support semantic knowledgebase is discussed in section 4. Section five reports on the research into complex-event processing aspects of the system, and section 6 concludes the paper and presents our plans for further work. 


\section{MotiVATION}

Ongoing investigation in our research group is involved in developing an Intelligent Inhabited Environment (IIE) infrastructure with the purpose of monitoring the activities of daily living of elderly occupants and assisting them in leading a good quality independent living [7]. The architecture of the IIE is illustrated in Figure 1, where the data collected from the sensor network are wirelessly communicated with a base station and eventually stored in a central database. The IIE relies solely on non-intrusive sensors as research has proven that the use of intrusive technology such as surveillance cameras for patient monitoring is generally not accepted [8]. Hence a combination of door/window entry point sensors, passive infrared motion sensors, and bed/sofa pressure sensors are utilised. The sensors produce long series of binary multidimensional data that are difficult to analyse and manipulate manually, so a combination of Principal Component Analysis and Fuzzy Rule-based Systems are applied to process the data and identify and also predict deviations from normal behaviour pattern [9], which are relayed to the health clinicians and the patient carer.

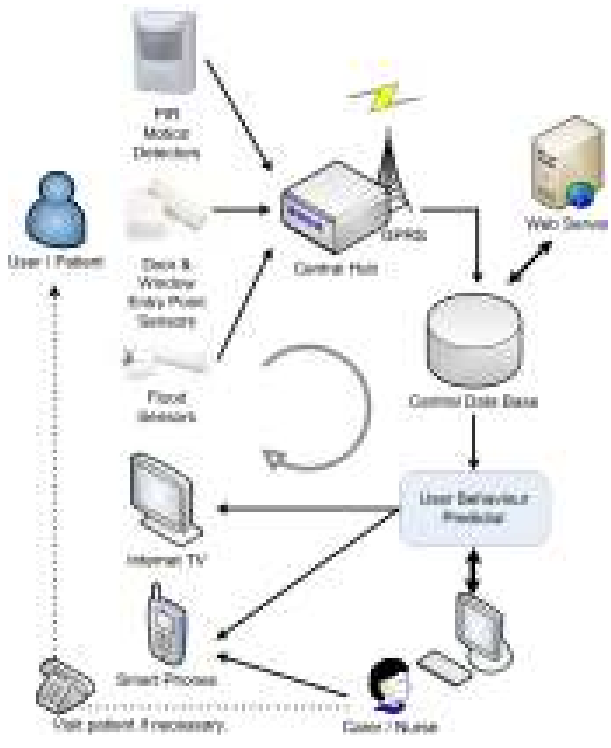

Figure 1. An overview of the IIE architecture

The research documented in this paper attempts to correlate the identified behavioural patterns specifically to the symptoms of dementia with the long term objective of developing a semantically-enabled dementia care decision support system (DCDSS) that can aid health clinicians in the remote assessment of the patients' condition, and thus reduce the demand for on-site care and support longer independent living for the dementia sufferers. To achieve this, the decision support software needs to not only posses a humanlike understanding of the complex concepts of the domains involved in a remote care system such as sensory movement information, human behaviour, disease diagnoses, and clinical guidelines, but also be able to intelligently interrelate these concepts to assist medical advice. Semantic web technologies are ideally suited to model such complex systems; In contrast to static terminology structures used for knowledge reference, semantic ontologies allow to describe at the taxonomy level the concepts and sophisticated relationships between the concepts (subsumption, cardinality, jointness, etc.), thus allowing for knowledge inference and reasoning. The ability to reason on the knowledgebase is particularly vital for the medical domain, where despite the often cleanly defined taxonomies, is characterised by the abundance of expert knowledge and a constantly growing and interacting number of clinical guidelines. Semantic knowledgebases are also inherently extensible [10] compared to traditional RDBS, which is again very useful for the ever-evolving medical domains.

\section{ConteXt-Aware SEMANTIC MODELling OF DEMENTIA CARE DeCision SUPPORT SySTEM}

Our approach to modelling the Dementia Care Decision Support System (DCDSS) is based on the premise that dynamic changes in the patients situational conditions (Context) such as room occupancy and movement within the living environment has to be analysed against the semantic knowledge about the condition' symptoms and the patient's related profile (medical history, age, etc.). Hence we can accurately describe our DCDSS as context-aware and knowledge-based. For instance context-awareness can detect short room occupancy and frequent movement between the rooms, which is analysed against the knowledge of the patient history of restlessness and inferred as deterioration in the patient's dementia condition.

It is worth reiterating that this effort aims to evaluate the feasibility of utilising the apparent advantages of semantic technologies in building a context-aware DCDSS rather than building a comprehensive prototype system. Hence, while we do research they dynamics of dementia care in published works and by interrogating the health clinicians, we suffice with minimal input that helps us to complete an implementation workflow cycle starting from capturing the patient's contextual data through sensors, feeding the results it into the ontology, then firing the reasoning engine to infer new knowledge based on various dementia criteria that was previously modelled and integrated into the ontology. Hence, we make the following assumptions about the system requirements:

1. The primary end-users of the system are health clinicians: doctors, nurses, and carers, who monitor the condition of the patient remotely.

2. The system should differentiate between alarming and critical anomalies in patient behaviour with critical anomalies being informed with a higher priority and in a way that demands quicker response.

3. The dementia patient is the sole occupier of the monitored residence. This significantly simplifies the movement and occupancy analysis.

4. The dementia residence consists of four rooms, a lounge, bedroom, kitchen, and bathroom with one main exit door. 
5. Although the living environment is equipped with a variety of non-intrusive sensors, in this study we investigate the output of the door entry point sensors (on/off switches), which relatively reliably indicate movement activities.

\section{4 BuILding the SEMANTIC KNOWLEDGEBASE}

\section{A. Knowledge Elicitation}

Knowledge elicitation refers to the process of gathering knowledge in terms of what concepts the domain experts understand to exist in a particular domain. One of the challenges facing ontology engineers is how to engage nontechnical domain experts in informing the ontology design without overwhelming them with the underlying technical complexities [11].

One of the primary knowledge sources we frequently referred to was Alzheimer's Association's '2010 Alzheimer's disease facts and figures [12] which has all currently known information pertaining to Alzheimer's Disease and Dementia. While this as well as other research publications was useful in gathering facts about the condition, it was important to consult with a medical expert who had the practical experience in managing the care for dementia patients, and who in addition to enriching our domain knowledge, represents the prime beneficiary of the envisaged DCDSS. A practicing psychiatrist who specialises dementia/ Alzheimer's Disease was interrogated to fulfil the knowledge-gathering phase.

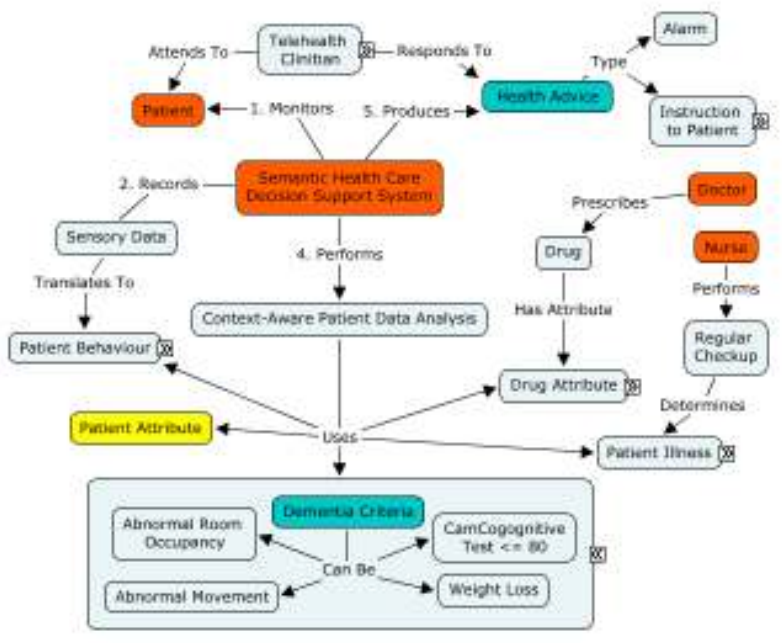

Figure 2. Concept Map

The gathered knowledge was conceptualised using a concept map as illustrated in Figure 2, which was later translated into formal ontology representation. The "Semantic Health Care DSS" uses various sensors to capture the room occupancy of the patient. Various sensors such as passive infrared sensors, contact sensors, location sensors, pressure sensors etc. would be used to determine the context of the patient. This live sensory information is recorded by the system and is translated into patient behaviour. The system then performs context-aware patient data analysis and produces health advice. Health advice could be in the form of alarms (for immediate attention), daily reports (for use by doctors for prescription) or even an instruction to the patient (such as reminders to take medicines, to sleep, to turn off the oven etc.). The healthcare clinicians respond to alarms generated by the system to attend to the patient immediately. The doctors use daily reports to review the prescription of drugs administered to the patient. The patient may or may not respond to the advice. In the latter case, an alarm would be generated to the healthcare clinicians for immediate attention.

\section{B. Ontology Engineering}

The concept map helped in the analysis of the application domain, which was key in encoding the identified entities and their interactions into a formal ontology language. Naturally we considered the reuse of publically available ontologies, particularly that the medical domain boasts a rich heritage of classified ontologies. There are efforts to build comprehensive taxonomies for the Dementia condition, notably SWAN [13], which is an interdisciplinary initiative to assemble information about neurodegenerative disorders such as the Alzheimer disease in a semantically structured format. The SWAN ontology is comprehensive and was developed primarily to promote the collaboration and exchange of biomedical information. Although we did use elements of the ontology to reference the dementia condition attributes in our ontology, the direct consumption of the ontology is beyond the scope of our current objectives as our primary concern in this effort is to investigate the feasibility of utilising the semantic technologies in building remote dementia care decision support systems rather than providing a comprehensive fully fledged solution at this juncture.

The ontology was divided into four main disjoint base classes (Figure 3). The first base class was created to store the patient details. Since AD/Dementia characteristics and the potential treatment depend on various factors such as the patient's age, the patient's ethnicity etc., we stored all these details in this base class of the ontology. This also allows us to create reports based on these various attributes of the patient in the future. Basic details such as the patient's name, ethnicity and age, the patient's location, etc. were also stored in this base class. Some of patient attributes such as ethnicity and age are elements of the knowledgebase involved in inferring anomalies in patient behaviour.

The next base class deals with the patient's medical history and the present medical condition of the patient. The most important entity of this section is the 'agreed patient behaviour'. This basically has configurable values that are to be used to determine abnormalities in patient behaviour. For example, values for normal bathroom usage for each patient have been defined using data type properties, which are later used to compare against current bathroom usage of the patient to determine abnormal bathroom usage. Object properties have been used to relate each patient to his/her 'agreed patient behaviour'. 
The third base class stores the live sensory data coming from the patients' homes. The live data from all patients go into this base class. This includes data such as the patients' live room occupancy data, the patients' motion pattern such as restlessness, accidental fall etc. This is the section of the ontology that contains temporal data. The information stored in this section is actually processed by an application, which calculates these values in a format that is understood by the ontology. This live data is compared against the patient's normal behaviour stored in the second section to determine anomalies in patient behaviour.

The fourth base class is the most important section of the ontology, which defines various criteria for classifying patient behaviour as normal, alarming or critical, but the actual logic that determines which patient behaviour is normal, alarming or critical needed to be implemented using semantic rules.

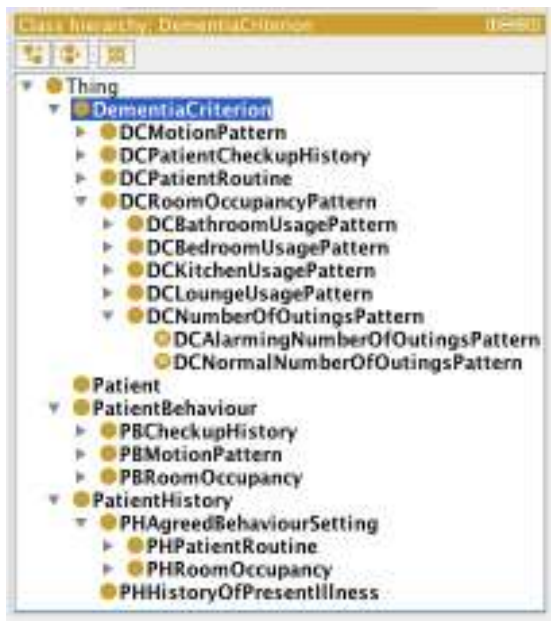

Figure 3. Fig. 3. Ontology Top-Level View

\section{The Reasoning Technology}

In contrast to reference-oriented semantic knowledgebase systems such as DBpedia [14] and GeoNames, knowledge inference technology is integral to the construction of context-driven knowledgebases. Initially we had to take into consideration how the classification of patient behaviour will be performed. There are two types of alarming symptoms of dementia. One is generic in nature such as high/low blood pressure reading, diabetes level etc. that are common to all patients. The other is specific to each patient such as the number of hours spent in the bathroom a day. The number of hours spent in the bathroom, for instance, could vary depending on the medical condition of the patient and the medicines the patient might be consuming amongst several others. Ideally, we would need to specify the normal bathroom usage in a placeholder that is variable in nature so that we could compare the actual number of hours the patient spends in a day against this variable. The problem is that the semantic reasoning engines responsible for classifying the ontology perform the classification in a single pass. Thus the necessary and sufficient conditions necessary to classify patient behaviour can contain only literals and cannot comprise variables such as a data type property. Hence, confining the ontology to basic semantic reasoning will result in creating a separate ontology for each patient that comprises hard-coded necessary and sufficient conditions. Clearly the development and maintenance overhead of such approach is unfeasible, and thus a rule-based system needs to be deployed to create generic ontology for the DCDSS.

We considered the utilisation of three rule systems: Jena built-in rules[15], SWRL[16], and Owlim [17] built-in rule system.

SWRL (Semantic Web Rule Language) is a proposal for a Semantic Web rules-language, combining sublanguages of the OWL Web Ontology Language (OWL DL and Lite) with those of the Rule Mark-up Language (Unary/Binary Data$\log$ ). Using SWRL allows us to compare two data type properties and then perform the classification. An example of the use of SWRL to encode our application rules is given below. This is a simple rule that expresses that if the bathroom usage of the patient is more than the ideal bathroom usage defined for the patient, then the bathroom usage should be classified as alarming.

hasOP PBBathroomUsage(?p, ?b), hasDP_PBBathroomUsage(?b, ?v1), hasDP ${ }^{-}$PHBathroomUsage(?h, ?v2), greaterThan(?v1, ?v2)

$->$

DCAlarmingBathroomUsagePattern(?b)

The syntax for the Jena rules encoding is very similar and equally satisfies our reasoning requirements as shown below

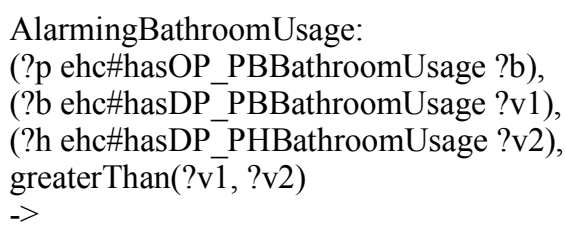

Owlim rules however do not support numerical evaluation necessary for our reasoning requirements, so we cannot encode $<$ math:greaterThan $>$.

The reasoning dynamics are exemplified by the analysis of the monitored behaviour of bathroom usage encoded in the rule above. As and when the patient uses the bathroom, an external software, the sensory output representing the total time spent in the bathroom is inserted as a triple in the ontology where it is linked to the corresponding patient through an object property. The SWRL rule associated to the monitored behaviour (exemplified above) evaluates the current bathroom usage against the patient's ideal bathroom usage, and if it is greater, it classifies the corresponding instance as a member of the class DCAlarmingBathroomUsagePattern during the inference process. 


\section{Initial Experimental Results}

Our initial experimentation only considered isolated events that give significant indication of the occupant's behaviour such as prolonged stay in rooms, restlessness (purposeless pacing between rooms), and uncharacteristic wandering outside the property. We obtained the simulated data of monitoring the activity of a varied number of patients for a day, and for each patient 520 significant sensory readings were injected into the ontology as instances representing their monitored activities, and then measured the reasoning time when inference was invoked. Deploying JenaTDB as the triple store running on a Mac mini (Intel Core 2 Duo $2.66 \mathrm{GHz} / 8 \mathrm{~GB}$ of memory) we obtained the results below for Jena and SWRL rule systems. The response time for inference is shown below.

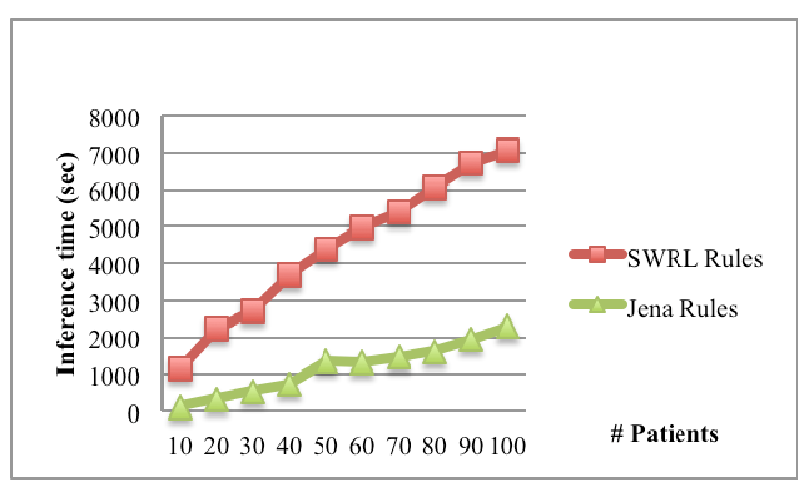

Figure 4. Inference time for SWRL and Jena Rules implementation

The inference time rises less exponentially for the Jena semantic rules and overall the response time is acceptable for the envisaged decision support system (less than 3 seconds for 100 patients. However, the complete implementation of a fully operational DCDSS will involve the processing of sensory output at high frequency as well as the analysis of the patient behaviour based on the processing of a multiplicity of atomic events (complex event processing).

\section{Event Processing in Remote HealthCare SYSTEMS}

A strategy to handle real-time events is critical to any decision support system, especially for health care where the responsiveness and accuracy of the system are critical to the patient's well being. For a semantic-driven system the first challenge in using ontology in a real-time system is with respect to when and how often the reasoning engine should be invoked. It is not feasible to invoke reasoning each time an event instance is inserted into the ontology, as that will overload the decision support system when large volumes of data are streamed from the sensors. Hence, reasoning has to be invoked at regular intervals that are continuously calculated in accordance with the current computational capacity of the system [18].

The second and more difficult challenge is Complex Event Processing (CEP) where abnormal behaviour is evaluated based on time-dependant occurrence and/or ordered occurrence of a number of events. We review next a number of contributions to the utilisation of CEP in semantic systems.

EP-SPARQL is an approach to Semantic Complex Event Processing that extends the SPARQL is a Resource Description Framework (RDF) query language with event processing and stream reasoning capabilities [19]. EPSPARQL extends SPARQL by adding the binary operators such as SEQ, EQUALS, OPTIONALSEQ and functions such as getDURATION(), getSTARTTIME() and getENDTIME to provide the capability of even ordering, a feature not supported by basic semantic rule systems such as SWRL. However, EP-SPARQL cannot handle situations where a variable number of unknown events need to be processed. In such case, it is impossible to write a predefined query since the events are not known in advance and also since they are variable in nature (in terms of the number of events that need to be processed). This can only be fulfilled by utilising a general purpose programming language at the API level on top of EP-SPARQL.

Kia Teymourian et al. in [20] propose an approach to ontology design that advises on complex events modelling rather than suggesting a CEP technology. Events can be described using a modular and a layered ontology model. As per this approach, there should be a shared top ontology to capture various concepts such as event, time, space, situation and other contextual-data. At the level below there would be additional specific domain, task and application ontologies that would contain more concrete or specialized concepts. Each domain would have its own set of events, which would be modelled as domain classes in the domain-specific ontologies each of which may be in relationship with other domain-specific ontologies. These domain-specific events can have object of data type properties associated with them. This level of modularity at the domain, task, and application levels certainly results in a better design and maintainability of the system especially when there are multiple domains, tasks and applications involved. Thus if there is a change in one of the applications, one needs to modify only the corresponding ontology and deploy it without affecting the rest of the ontologies. This improves the overall maintainability of the system. Although this approach is useful when there are multiple domains and applications accessing the ontologies, this may result in unnecessary processing overhead for single domain/single application systems such as dementia.

The effort in [21] proposes a generic architecture for processing complex events using semantic technology. The framework defines five different components each of which may be replaceable for different applications: A user interface to visually build complex event definitions, a semantic event middleware to process complex event definitions a management module interfaces for programming and accessing different types of sensors from different sources, an event ontology that stores the models of sensor networks, complex events etc., and a CEP server that will determine complex events from multiple data streams. This is a useful contribution that aids in designing semantic frameworks that require the utilisation of complex event processing technology. 
Complex Event Processing using Semantic Technologies is an evolving area of research. Semantic rules such as SWRL can be seamlessly integrated into the semantic ontologies but do not support the event workflow capabilities of external Event Processing languages such as EPSPARQL, which on the other hand add to the complexity of building the decision support system. Our next task is to weigh the advantages of both approaches and also consider the deployment of an external proprietary events' preprocessor that can handle the temporal attributes of events outside the semantic knowledgebase; that however would be at the expense of the generic reuse of the developed system.

\section{CONCLUSIONS AND FURTHER WORKS}

In this paper, we reported on our investigation into the feasibility of utilising semantic technologies in decision support for remote Dementia care. We explain how our approach to context-aware modelling facilitates interfacing the output of non-intrusive sensors, which that monitor the patient behaviour in the patient's residence, to the semantic knowledge about the patient's condition and the related medical advice. Our research identifies that the reasoning process is the critical link in the semantic-driven decision support system. Therefore, our effort carefully analyses published works in the area, and undertakes relevant experiments testing the suitability of the underlying implementation technologies, with the purpose of establishing the requirements for building an knowledge inference engine, which is capable of efficient processing of the complex events that represent the patient's behaviour and subsequently offer support in delivering the relevant medical advice. The next phase of this work involves the completion of the system implementation by integrating a complex events processor into the semantic knowledgebase and conducting field trials.

\section{REFERENCES}

[1] Garry Young: The implications of an ageing population on the UK economy [online]. Available at: https://www.bankofengland.co.uk/publications/workingpapers/wp159 .pdf (last accessed: 14/01/12)

[2] Mark W J Strachan, Jacqueline F Prince, Brian M Frier: Diabetes, cognitive impairment, and dementia [online]. http://www.ncbi.nlm.nih.gov/pmc/articles/PMC2174783/ (last accessed: $14 / 01 / 12$ )

[3] Carl A Thompson, Karen Spilsbury, Jill Hall, Yvonne Birks, Colin Barnes, Joy Adamson: Systematic review of information and support interventions for caregivers of people with dementia [online]. Available at: http://www.biomedcentral.com/1471-2318/7/18 (last accessed: 14/01/12)

[4] Christian Bakker et al. Needs in Early Onset Dementia: A Qualitative Case From the NeedYD Study. AM J ALZHEIMERS DIS OTHER DEMEN December 2010 vol. 25 no. 8 pp. 634-640 (2010)

[5] Just Checking dementia support system. http://www.justchecking.co.uk/ [accessed: 12/1/2012]

[6] Meiland, F, Reinersmann, A, Bergvall-Kareborn, B, Craig, D, Moelaert, F, Mulvenna, Maurice, Nugent, CD, Scully, T, Bengtsson, J and Dröes, R.-M: COGKNOW: Development and evaluation of an ICT-device for people (2007)
[7] A. Lotfi, C. Langensiepen, S. M. Mahmoud, M. J. Akhlaghinia: Smart homes for the elderly dementia sufferers: Identification and prediction of abnormal behaviour, Journal of Ambient Intelligence and Humanized Computing. doi:10.1007/s12652-010-0043-x. (2011)

[8] A. Kenner. Securing the elderly body: Dementia, surveillance, and the politics of aging in place. Surveillance and Society, 5:252-269 (2008).

[9] Sawsan M. Mahmoud, Ahmad Lotfi and Caroline Langensiepen: User Activities Outlier Detection System Using Principal Component Analysis and Fuzzy Rule-Based System, Fifth International Conference on PErvasive Technologies Related to Assistive Environments, Crete, Greece (submitted 2012)

[10] Asunción Gómez-Pérez, Mariano Fernández-López, Oscar Corcho: Ontological engineering: with examples from the areas of knowledge management, e-commerce and the Semantic Web. Springer, 2004 Business \& Economics with mild dementia. In: Medical and Care Compunetics 4. (Eds: Bos, L and Blobel, B), 978-I-58603-751-2, pp. 166-177 (2004).

[11] Osman T., Thakker D., Nathan M.: Research Notes on the Practical Deployment of Semantic Knowledge Bases. Proceedings of the 12th European Conference on Knowledge Management ECKM 2011 Passau, Germany. Vol.1 pp. 737-745, ISBN: 978-1-908272-106-5, September 2011. (2011)

[12] Alzheimer's Association, 2010 Alzheimer's Disease Facts and Figures, Alzheimer's \& Dementia, Volume 6. (2010)

[13] Paolo Ciccarese, Elizabeth Wu, Gwen Wong, Marco Ocana, June Kinoshita, Alan Ruttenberg, Tim Clark: The SWAN biomedical discourse ontology. Journal of Biomedical Informatics, Volume 41, Issue 5, October 2008, Pages 739-751 (2008)

[14] Auer, S.,Bizer, C., Cyganiak, R., Ives, Z.: DBpedia: A Nucleus for a Web of Open Data. In: 2nd Asian Semantic Web Conference. Springer Berlin / Heidelberg (2007)

[15] Jena 2 Inference Support http://jena.sourceforge.net/inference/ (last accessed 3/3/2012)

[16] SWRL: A Semantic Web Rule Language http://www.w3.org/Submission/SWRL/ (last accessed 3/3/2012)

[17] Kiryakov, A.: OWLIM: balancing between scalable repository and light-weight reasoner. In:Developer's Track, WWW2006 (2006).

[18] Osman, T, Bargiela, A: FADI: A Fault-Tolerant Environment for Open Distributed Computing, IEE Proceedings on Software, Vol. 147(3), pp 91-99. (2000)

[19] Darko Anicic, Paul Fodor, Sebastian Rudolph, Nenad Stojanovic: EP-SPARQL: A Unified Language for Event Processing and Stream Reasoning. Proceedings of the 20th International Conference on World Wide Web, WWW 2011, Seiten: 635-644, ACM (2011)

[20] Kia Teymourian, Olga Streibel, Adrian Paschke: Towards Semantic Event-Driven Systems. New Technologies, Mobility and Security (NTMS), 2009 3rd International Conference, pp 1-6. (2009)

[21] Kerry Taylor, Lucas Leidenger: Ontology-Driven complex event processing in heterogeneous sensor networks. Lecture Notes in Computer Science. Volume 6644/2011, 285-299. (2011) 